\title{
Suffiziente Haushalte - Illusion oder Möglichkeit?
}

Ursprünglich veröffentlicht in: uwf UmweltWirtschaftsForum, 2015, Jg. 23, Nr. 1-2, S. 15-21

Die endgültige Publikation ist erhältlich bei Springer via http://dx.doi.org/10.1007/ so0550-015-0348-5 


\section{Suffiziente Haushalte - Illusion oder Möglichkeit?}

* Autorin:

Melanie Lukas

Wuppertal Institut für Klima, Umwelt, Energie

Döppersberg 19

42103 Wuppertal

E-Mail: melanie.lukas@wupperinst.org

Tel.: +49 202 2492-302

Fax: +49 202 2492-138

Dies ist die begutachtete, akzeptierte Manuskriptversion des Artikels. Aus dem Veröffentlichungsprozess können nachträgliche Änderungen resultieren, die sich z. B. auf die Formatierung und Zeichensetzung auswirken. Derartige Änderungen sind hier nicht berücksichtigt. Die endgültige Version wurde in der oben genannten Zeitschrift veröffentlicht. 


\section{Suffiziente Haushalte - Illusion oder Möglichkeit?' Melanie Lukas}

Schlüsselwörter: Suffizienz, Haushalt, Konsum, nachhaltige Entwicklung, Verbraucherforschung

\section{Einstieg}

Der folgende Beitrag widmet sich der Frage, wie suffizient private Haushalte im heutigen Wirtschaftssystem agieren können und welche Grenzen sich offenbaren. Private Haushalte sind neben den Unternehmen, staatlichen Institutionen und intermediären Organisationen eine zentrale Leistungsinstanz für die Produktion von Gütern und Dienstleistungen und nehmen damit die Versorgung der Menschen ein (Götz et al., 2011; Liedtke et al., 2014). Haushalte bieten viel Potential für die Verankerung von Suffizienzstrategien, trotzdem gibt es gewissermaßen genauso viele Herausforderungen und Barrieren rund um diesen Themenschwerpunkt. Die Vision dabei: Durch suffizienteren Konsum könnten Haushalte in ihrem privaten Entscheidungsraum langfristig zukunftsfähiger agieren. So würde der haushaltsspezifischer Ressourcenverbrauch gesenkt und suffizientere Unternehmenslösungen würden durch ausgewählte Konsumentscheidungen von Haushalten unterstützt werden. Von dieser Vision ist die Gesellschaft aktuell aber zugegebenermaßen immer noch weit entfernt. Trotzdem erscheint es wichtig, sich diesem Thema empirisch zu nähern, insbesondere um Potentiale und Herausforderungen eines suffizienteren Handelns in den heutigen Haushaltsund Gesellschaftsstrukturen zu skizzieren. Die Argumentation des Beitrags fußt deshalb auf den Ergebnissen aus 42 qualitativen, problemfokussierten Interviews mit Verbraucherinnen und Verbrauchern in ganz Deutschland.

Der Beitrag schließt mit einem Fazit und skizziert die Antwort auf die Frage, ob und wie Haushalte suffizient sein können und welche Herausforderungen sich für die Zukunft ergeben.

\footnotetext{
${ }^{1}$ Die hier präsentierten Ergebnisse wurden im Rahmen des Dissertationsprojekts der Autorin erarbeitet.
} 


\section{Hintergrund - Der Haushalt als Formgeber für Konsumentscheidungen}

Haushalte werden durch ihre Mitglieder zu agierenden Institutionen, deren Hauptfunktion die unmittelbare Bedarfsdeckung ist, d.h. die Organisation von Produktions- und Konsumprozessen in der gesamtwirtschaftlichen Güterformation (Kettschau et al., 2000; Spangenberg \& Lorek, 2002). Dafür müssen Geld, Arbeit und Ressourcen eingesetzt und umverteilt werden (Schlegel-Matthies et al., 2009). Es handelt sich um einen metabolischen Prozess, der die Zufuhr, Transformation und Abfuhr von Materie und Energie einschließlich Informationen und Austauschbeziehungen mit der sozioökonomischen und ökologischen Umwelt erfordert (Piorkowsky, 2000). Haushalte sind somit als private Wirtschaftseinheiten und gleichzeitig als kulturelle Räumen wahrzunehmen, in denen Konsum erlernt und geprägt wird.

Der Wandel der Gesellschaft hin zu einer Erlebnis- und Konsumgesellschaft unterstützt die Wertschätzung für den materiellen Konsum (Schulze, 2005) und damit auch die Abhängigkeiten der Haushalte von den Strukturen des Wirtschaftsraums. Durch ein hohes $\mathrm{Ma} ß$ an Erwerbstätigkeit aller erwachsenen Haushaltsangehörigen, ist auch ein hohes Maß an Fremdversorgung zur Normalität geworden. Die Haushalte sind auf ein notwendiges Konsumniveau angewiesen und stehen somit in enger Wechselwirkung mit Unternehmen. Parallel dazu hat der Ausstattungsgrad mit Haushaltsgütern in den privaten Haushalten rapide zugenommen. Heute sind 99\% der Haushalte mit einem Kühlschrank ausgestattet, 94\% mit einem Fernsehgerät und 77\% haben mindestens einen PKW (Glatzer, 2013). Infolgedessen hat sich der umweltrelevante Fußabdruck eines Haushalts verschlechtert (Lettenmeier et al., 2014). Wie es Fischer und Sommer (2011) verdeutlichen, bewegen wir uns heute innerhalb eines Haushalts nicht mehr nur in einem von Grundbedürfnissen gesättigten Raum, denn diese sind bereits gesättigt. Der Haushalt wird somit vom Wirtschaftsort mit Erholungsfunktion zum Erholungsort mit eigener Wirtschaft. Infolgedessen ergibt sich die Frage, in welcher Form Haushalte überhaupt suffizient handeln können und welche Hemmnisse und Perspektiven sich für Haushalte als auch für die Beziehung zwischen Haushalten und Unternehmen ergeben.

\section{Suffizienz - (k)ein Thema in Haushalten?}

Soll der Suffizienzgedanke in den Konsumalltag des Privathaushalts integriert werden, ergibt sich häufig das Problem der Abgrenzung eines suffizienten Handelns von regulären Handlungsabfolgen und Praktiken. Die Übergänge zwischen konventionellem und 
suffizientem Konsum erscheinen häufig fließend. Sozialwissenschaftlich gesehen ist ein suffizientes Handeln grundsätzlich in allen möglichen Alltagspraktiken denkbar (Bittlingmayer, 2000:106). Die detaillierte Beschreibung suffizienter Praktiken im Haushalt ist somit häufig eine größere Herausforderung, als es erscheinen mag.

Die 42 Interviews $^{2}$ mit jeweils einem Haushaltsoberhaupt oder -mitglied offenbaren Ansatzpunkte für suffizienteres Handeln, und dass obwohl im Rahmen dieser qualitativen Exploration keine „Aussteiger-Haushalte“ interviewt wurden. Insgesamt lassen sich in sieben Interviews suffizienz-orientierte Handlungskonzepte erkennen, die von Personen ausgeübt werden, die sich selbst als ,umweltbewusst“ und „sozial-/gemeinwohl-orientiert“, aber nicht unbedingt als „sparsam“ oder „suffizient“ beschreiben. Diese Personen arrangieren sich mit den konsumgesellschaftlichen Strukturen und agieren in diesen Strukturen meist nach einem individuellen Überzeugungsschema,. Trotzdem konsumieren die Personen und wenden sich nicht von der Außenwelt ab. Diese erste Verortung ist wichtig und führt zu der Annahme, dass Suffizienz in Haushalten wie folgt $\mathrm{zu}$ definieren ist: Suffizienzstrategien in der Konsumgesellschaft kommen nicht ohne Konsumtätigkeiten aus. Es dreht sich also nicht um Alternativen zum Konsum, sondern um alternative Formen von Konsum sowie um ausgewählte Verzichtshandlungen, wenn es um die Definition einer haushaltsnahen Alltagssuffizienz geht. Dies schließt zukünftig eine neue Beziehung zwischen Unternehmen und Haushalten ein. Alternative Formen des Konsums werden immer noch viel zu häufig von Unternehmen ausgeblendet und es wird suggeriert, dass lediglich neuwertige Konsumgüter distinktionsfähig, nützlich und brauchbar sind. Unter diesem Blickpunkt stellt sich die Frage, wo und wie Haushalte in einer Konsumgesellschaft überhaupt affin für Suffizienzstrategien sind, denn eine Konfrontation mit suffizienteren Handlungsstrukturen ist in der Konsumgesellschaft nahezu ausgeschlossen. Grundlegend wird dabei die Definition von Stengel (2011:130) herangezogen und in etwas abgewandelter Form berücksichtigt:

Die Suffizienzstrategie impliziert die Reduktion von ökologischen [und sozialen] Auswirkungen des eigenen Handelns und führt damit zu einer Reduktion bzw. einem Verzicht auf Dienstleistungen oder Produkte mit hoher Ressourcenintensivität, aber auch zu einer Modifikation von Praktiken.

Daraus lässt sich ableiten: Suffizienz in Haushalten findet auf der Ebene der Praktiken statt und wird durch Alternativhandlungen - wie Tauschen, Reparieren, Teilen etc. umgesetzt. Darüber hinaus sind einige Verzichtsstrategien $\mathrm{zu}$ erkennen, die aber während des Handlungsvorgangs von den Personen selbst nicht als Verzicht empfunden werden. Diese

\footnotetext{
${ }^{2}$ Die Interviews wurden mit Hilfe der Grounded Theory Methodologie (Strauss \& Corbin, 1996) ausgewertet.
} 
Definition stellt die Beziehung zwischen Haushalten und Unternehmen zwar nicht in Frage, beinhaltet aber, dass sich Unternehmen in einigen Bereichen von einer Art Emanzipation der Haushalte ausgehen können, je suffizienter die Lebensarten gestaltet werden, desto mehr verändert sich die Beziehung beider Wirtschaftseinheiten. Die Alternativhandlungen könnten u.a. eine Neuordnung von Geschäftsmodellen beinhalten, denn durch suffizientere Konsumlösungen können Haushalte an einigen Schnittstellen neue Bedarfe entwickeln, z.B. nach Reparatur-, Tausch- oder Sharing-Dienstleistungen. Und auch wenn es bereits eine breite Auswahl von Dienstleistungen gibt, scheinen die Möglichkeiten nicht ausgeschöpft. Insbesondere dann, wenn mehr und mehr Haushalte suffizient leben. Trotzdem stehen diesem suffizienteren Handeln viele Hindernisse entgegen. Das folgende Kapitel präsentiert deshalb eine Auswahl der empirischen Erkenntnisse rund um die gesellschaftlichen und ökologischen Potentiale eines suffizienteren Handelns von Haushalten in der heutigen Konsumgesellschaft.

\section{Empirische Ergebnisse - Vielfalt und viel Potential}

\section{Was impliziert ein suffizienteres Handeln?}

Bei der empirischen Untersuchung der Suffizienzstrategien in Haushalten wird deutlich: Das Suffizienzkonzept im Privathaushalt basiert auf der Umsetzung veränderter Kulturtechniken in Form sozialer Praktiken in möglichst allen haushaltsnahen Konsumbereichen wie Mobilität, Ernährung, Bauen/Wohnen sowie im Konsum- und Freizeitverhalten. Die Prämisse der Reduktion ökologischer und sozialer Negativauswirkungen sollte dabei die Handlungen regieren, ohne einem normativen Duktus zu verfallen.

Die suffizienz-orientierten Haushalte weichen bei der Umsetzung suffizienterer Praktiken in Teilen von heute gängigen Konsumpraktiken ab, ohne sich dabei aber einer vollkommenen Umstellung der Alltagspraktiken zu unterziehen und ohne sich dabei als ,asketisch“ oder „sparsam“ zu bezeichnen. Das heißt, die Teilnehmer sind nicht per se suffizient, sondern handeln in Teilbereichen ihres Lebens suffizient. Oder vereinfacht gesagt, dort wo die einzelnen Personen es als einfach empfinden, suffizienter zu handeln, dort wird ein Handeln umgesetzt. Diese subjektive Einschätzung ist aber von Situation zu Situation variabel. Wenn in einem Haushalt suffizienter gehandelt wird, dann wird häufig nicht vollkommen auf „etwas verzichtet", sondern es wird nach Alternativlösungen gesucht oder es werden Praktiken verändert. Dabei regieren individuelle Prämissen u.a. Gesundheit, Sparsamkeit oder 
finanzieller Restriktion aus denen sehr individuelle Denkschemata diffundieren, wie das Beispiel der individuellen Mobilitätsstrategie von Sabine verdeutlicht:

[...] Priorität ist erstmal Fahrrad fahren, dann Bahn und Bus und dann Auto. (Sabine - 00:18:26-1)

Sabine bringt es in ihrem Interview auf den Punkt. Die meisten suffizienz-orientierten Personen versuchen nach einem sogenannten Schema der Relation zu handeln. Diese Denkschemata sind individuell auferlegt und werden nicht als beengend empfunden. Es wird eine individuelle Strategie zur Bewältigung des Alltags entworfen, eine Art imperative Selbstbindung. Entscheidungsmuster werden reflektiert und kontinuierlich angepasst, Alternativen werden genutzt, alle Möglichkeiten umweltbewusster oder sozialorientiert im Alltag zu handeln werden möglichst konsequent ausgenutzt. Sabine bevorzugt das Fahrrad, weil sie gern draußen unterwegs ist und somit sich selbst und der Umwelt etwas „Gutes tut“. Dabei erscheint es interessant, dass vor allem das eigene ,gute Gewissen“ im Vordergrund steht und somit immer der eigene individuelle Nutzen für den Einzelnen fokussiert wird. Das Thema Sparsamkeit oder Verzicht tritt dabei nicht in Erscheinung. Durch diese subjektive und selbst auferlegte Bindung wird das Handeln selbst kaum als anstrengend oder negativ assoziiert - gleichzeitig kann aber flexibel von der eigenen favorisierten Tätigkeit abgewichen werden, wenn die Situation ein verändertes Handeln verlangt. Ein wichtiger Ankerpunkt ist also die Kombination zwischen der eigenen Selbstbindung und der Flexibilität. Die Person versucht daher in ihrem Handlungsraum möglichst die „besten Alternativen“ zu finden.

Diese idealtypische Selbstbindung lässt sich bei den weniger suffizienz-orientierten Haushalten und damit bei der Mehrzahl der untersuchten Haushalte nicht feststellen. Trotzdem wird bei deren Untersuchung deutlich, dass diese häufiger als Ihnen bewusst ist, suffizient handeln. Nämlich dann, wenn Zeit oder Budgetrestriktionen ein konkret suffizientes Handlungsschema verlangen oder wenn sich ein individueller Zusatznutzen einstellt. So werden sich in Mobilitätsfragen nicht wenige Personen ebenfalls für die Nutzung des Fahrrads entscheiden, wenn u.a. kurze Wege zurückzulegen sind, sich dadurch Zeitersparnisse ergeben und gleichzeitig noch der Gesundheitsnutzen bewusst ist. Die Umweltorientierung steht dabei an hinterer Stelle.

Unabhängig von der Affinität zur Suffizienz wird bei der Untersuchung der Personenhaushalte deutlich, dass die Praktiken täglich reversibel sind und sich situativ - in Abhängigkeit von Zeit, Geld und Struktur - unbewusst suffizienz-orientierte Handlungsmuster ergeben können. 
Die empirischen Erkenntnisse zeigen zudem, dass die Struktur und die Ausstattung der Haushalte wichtige Faktoren für die Entscheidungsfindungen in vielen Konsumbereichen sind. Hier spielen vor allem die Fragen, ob die Person selbstversorgend ist oder in einer Gemeinschaft lebt und wie diese Gemeinschaft beschaffen ist, eine entscheidende Rolle. Denn der Haushalt als Setting für Konsumsozialisation ist ein wichtiger Ort, suffizientes Handeln in die Tat umzusetzen, z.B. bei der gemeinsamen Ernährungszubereitung und beim Verzehr. Will eine Person ökologisch-orientiert im Haushalt agieren, kommt es meist zur direkten oder indirekten Beeinflussung der Mitglieder des Haushalts. So beschreiben viele der Haushaltsmitglieder, dass in ihrem Haushalt häufig eine Person ,sich irgendwie mehr mit solche Sachen wie Umweltschutz etc. beschäftigt“. Damit werden unterschiedliche Reihungen von Konsumentscheidungen an diese Person abgetreten. Hier scheint dann die Haushaltshierarchie entscheidend. Ist eine Person in der Haushaltshierarchie an höherer Stelle zu verorten, ist diese Person der Knotenpunkt für die Entscheidungsstrukturen des Haushalts. Dieser Sachverhalt führt $\mathrm{zu}$ einem weiteren Aspekt, der die Wechselwirkung unterschiedlicher Konzepte einer Transformation verdeutlicht: Suffizienz wird im Haushalt häufig durch Konsistenz- und Effizienzmaßnahmen unterstützt - Beispiel: Nutzung von Ökostrom (Konsistenzmaßnahme) und gleichzeitige Reduktion des Stromverbrauchs durch die Veränderung von Haushaltspraktiken und die Unterstützung dieser durch eine entsprechende Infrastruktur (Effizienzmaßnahme), z.B. durch energiesparende Steckdosen mit Schalter.. Suffiziente Handlungsmuster isoliert zu betrachten ist also nahezu unmöglich und gleichzeitig wenig system-orientiert.

Ähnliche Ergebnisse zeigt auch eine Untersuchung in Schweizer Haushalten (Bruppacher, 2008), die noch einen weiteren Aspekt aufzeigt. Dort wird zwischen einmaligen Entscheidungen und Aufwendungen, Routinen und sinnstiftende Tätigkeiten unterschieden. Investitionen sind häufig mit einer Einmal-Entscheidung verknüpft und haben meist einen direkten Nutzen in Form von Zeit- oder Geldersparnissen. Routinen, z.B. Licht- und Stromsparmaßnahmen, die nach einiger Zeit von den Haushaltsmitgliedern nicht mehr hinterfragt werden, werden irgendwann kaum noch wahrgenommen und hängen eventuell auch mit einer Zeit- und Kostenersparnis zusammen. Die sinnstiftenden Verhaltensmuster, wie die Veränderung der Mobilitätsentscheidungen oder die Reduktion des Fleischkonsums werden dagegen von weniger suffizienz-affinen Haushalten eher als unangenehm empfunden und das scheint entscheidend für die Suffizienzverortung. Wie es auch Scholl (2009) beschreibt, ist davon auszugehen, dass neue suffizientere Praktiken, auch wenn sie nicht 
vollkommen von der Norm der Gesellschaft abweichen, erst einmal ungewohnt erscheinen. Der Weg hin zu einer Routine in diesen Bereichen erscheint sehr viel schwieriger und so dauert es auch einige Zeit, bis diese Praktiken in ein Routineverhalten übergehen können. Die suffizienz-orientierten Haushaltsmitglieder haben diese Art einer Transformation bereits vollzogen und empfinden ihr Handeln nicht mehr als umständlich, reduktiv oder unangenehm. Die anfänglichen Hürden sind also in der Regel nach der Etablierung der Routine nicht mehr vorhanden.

Zudem wartet eine weitere Herausforderung bei der Umsetzung suffizienterer Praktiken. Dies kann untermalt werden durch das folgende Interviewzitat einer Teilnehmerin:

Man kann mit Bio-Lebensmitteln auch sehr günstig kochen, man kann nur nicht genauso wie immer kochen wie es vorher war. Stück Fleisch, Gemüse etc. - Das Essen ist viel teurer. Wenn man aber sich von mehr Getreide etc. ernährt wird es günstiger, wenn man umdenkt ist es nicht teurer. (Marie - 188 // 00:37:22-1)

Dieses Beispiel von Marie steht stellvertretend für viele Transformationsprozesse im Haushalt. Denn häufig führt ein Umdenken hin zu einem suffizienteren Handeln zu einer Kette von Veränderungen. An diesem Beispiel verdeutlicht sich: in dem Moment, in dem im Haushalt suffizienter gegessen wird, werden häufig die gesamten Essgewohnheiten Schritt für Schritt umgestellt. Und auch wenn diese Umstellung nicht als negativ empfunden wird, so erfordert diese Umstellung ein gewisses Durchhaltevermögen und eine gewisse Unterstützung des sozialen Umfelds. Generell wird im Bereich Ernährung meist in suffizienteren Haushalten vegetarisch gespeist, es muss aber nicht komplett auf Fleisch oder Fisch verzichtet werden. Häufig wird auf Wochenmärkten oder in Bioläden eingekauft. Allerdings werden von der Mehrheit der Haushalte die Bereiche, in denen ständig neue sinnstiftende Entscheidungen zu treffen sind, häufig fortwährend als unangenehm empfunden. Meist entsteht dann bei diesen Haushalten eine sehr konkrete Veränderungsresistenz (dazu auch: Bruppacher, 2008). Diese Veränderungsresistenz wird häufig durch infrastrukturelle, zeitliche oder bugdet-restriktive Bedingungen untermauert und festigt sich ebenfalls durch die Haltung des sozialen Umfelds.

Die Unterstützung des sozialen Umfelds ist somit als weiterer Einflussgeber auf der MetaEbene zu erläutern, insbesondere dann, wenn ein sichtbarer und konkreter Verzicht verübt wird. Aktive Verzichtsmotive, die im Rahmen der gesellschaftlichen Orientierung weniger akzeptiert sind, werden nicht selten zu einer Barriere für Veränderung. Konkret lässt sich das am Beispiel des aktiven Verzichts auf einen eigenen PKW veranschaulichen: Markus beschreibt sich selbst als „militant“ in Bezug auf den Verzicht auf einen eigenen PKW. Er 
sieht keine Notwendigkeit für ein Auto in der Stadt und lehnt den Besitz eines eigenen Autos auch aus seinem ökologischen Bewusstsein heraus ab. Dabei stößt er in seinem direkten sozialen Umfeld - in Form seiner Frau und seinen zwei Kindern - auf Zustimmung, erntet aber im erweiterten sozialen Umfeld - hier: seine Mutter - Unverständnis. Denn sogar das Geschenk der Mutter in Form eines PKWs lehnt er ab. Im noch breiteren sozialen Umfeld Schulfreunde der Kinder - stößt seine Haltung sogar auf Verwunderung.

Ich habe schon oft gemerkt, so in der Diskussion gegenüber den anderen, also naja "komische Familie, die haben kein Auto" und so... (Michael - 00:37:04 // 80)

Bei Markus sind verschiedene Perspektiven festzustellen, mit denen Individuen konfrontiert sind, wenn ein aktiver Verzicht eines gesellschaftlich voll akzeptierten Produkts ausgeübt wird oder eine Praktik abgelehnt wird - drei unterschiedliche Phänomene sind festzuhalten (Tabelle 1):

Tabelle 1: Übersicht zu Reaktionen des sozialen Umfelds auf Verzichtsstrategien (eigene Darstellung)

\begin{tabular}{|c|c|c|}
\hline Phänomen & Erklärung & $\begin{array}{l}\text { Konsequenz/ } \\
\text { Beeinflussung }\end{array}$ \\
\hline $\begin{array}{l}\text { Abweichen von } \\
\text { Haushalts-Konformität }\end{array}$ & $\begin{array}{l}\text {... wenn das direkte tägliche soziale Umfeld } \\
\text { (Familienhaushalt, Wohngemeinschaft etc.) } \\
\text { mein Tun akzeptiert bzw. unterstützt. }\end{array}$ & hoch / hoch \\
\hline $\begin{array}{l}\text { Abweichen von } \\
\text { Gruppen-Konformität: }\end{array}$ & $\begin{array}{l}\text {... wenn das erweiterte soziale Umfeld } \\
\text { (Familie, Freunde etc.) mein Tun nicht } \\
\text { akzeptiert bzw. unterstützt. }\end{array}$ & mittel / mittel \\
\hline $\begin{array}{l}\text { Abweichen von } \\
\text { Gesellschafts- } \\
\text { Konformität: }\end{array}$ & $\begin{array}{l}\text {... wenn das erweiterte soziale Umfeld } \\
\text { (Familie, Freunde etc.) das Tun nicht } \\
\text { akzeptiert bzw. unterstützt. }\end{array}$ & gering / mittel-gering \\
\hline
\end{tabular}

Die Tabelle 1 erläutert die drei möglichen Konformitäts-Abweichungen, die im Rahmen von Verzichtsstrategien erfahren werden. Personen, die auf etwas verzichten oder „etwas anders machen“, weichen von der gesellschaftlichen Konformität ab. Die gesellschaftliche Konformität beeinflusst die Personen dabei natürlich weniger als die Haushaltskonformität. Die Haushaltskonformität muss nahezu bei allen suffizienz-orientierten Praktiken gegeben sein, denn alle Haushaltsmitglieder müssen direkt oder indirekt diese Praktiken mittragen. Darüber hinaus sind Freunde und Familienkreis nicht selten auch von veränderten Praktiken betroffen. Dadurch können sich Konflikte ergeben, die in Form eines Unverständnisses für den Einzelnen oder für den gesamten Haushalt geformt werden. Die fehlende gesellschaftliche Konformität trifft dann ein, wenn Suffizienzstrategien sehr deutlich für 
Außenstehende sichtbar sind, wie hier im Beispiel durch den aktiven Verzicht auf ein Auto im Haushalt.

Ein interessanter Vergleich erschließt sich, denn ähnlich kann es auch Personen ergehen, die Fasten oder Diät halten, bzw. andere Genussmittel der Gesellschaft ablehnen. Diese Personen werden - unabhängig von einer gesonderten Orientierung - nicht selten in ihrem eigenen Umfeld damit konfrontiert, dass ihre Praktik von der Norm ,abweicht“ (dazu auch: Cherrier \& Gurrieri, 2012). Diese Konformität mit dem sozialen Umfeld ist für den Einzelnen sehr entscheidend, insbesondere dann, wenn seine eigene Überzeugung zum suffizienteren Handeln noch nicht endgültig gefestigt ist. Aus dem Interviewsample heraus lassen sich zudem insbesondere die Angst vor Komfortverlust und die eigene Bequemlichkeit als starke Barrieren erkennen, weshalb einfache und meist kleine Veränderungen, die bereits ein suffizienteres Haushaltsleben gestalten könnten, nicht umgesetzt werden.

Neben der sozialwissenschaftlichen Erschließung der Haushaltssituation drängt sich eine beispielhafte Untersuchung der ökologischen Relevanz suffizienter Handlungsweisen auf und somit die Frage:

\section{Welches ökologische Potential steckt in einem suffizient handelnden Haushalt?}

Diese Frage soll mit Hilfe zweier sehr kontrastreicher Interviews beantwortet werden. Dazu wurden ein konventionell handelnder Haushalt und ein sehr suffizient ausgerichteter Haushalt gegenüber gestellt ${ }^{3}$. Die Ergebnisse werden mit Hilfe des Material Footprints ${ }^{4}$ ausgedrückt.

\footnotetext{
${ }^{3}$ Die hier vorgestellte Beispielrechnung basiert auf den Angaben aus zwei Haushaltsinterviews, die im Rahmen des vorliegenden Beitrags dann beispielhaft auf einen 2-Personen-Haushalt umgerechnet worden sind. Mit Hilfe einer ergänzenden Befragung dieser ausgewählten Haushalte wurde rekonstruiert, dass bspw. in einem konventionellen Haushalt zwei Autos vorhanden sind, während sich z.B. der suffiziente Haushalt ein Auto mit anderen Parteien teilt und das zum Beispiel ähnliche Gewohnheiten im Bereich Ernährung und Freizeit bei einem 2-Personen-Haushalt vorliegen.

${ }^{4}$ Material Footprint: Basierend auf der MIPS-Methode zur Ermittlung von Ressourcen In- und Outputs, (siehe Lettenmeier et al. 2009 http://www.econstor.eu/bitstream/10419/59292/1/716261502.pdf)
} 


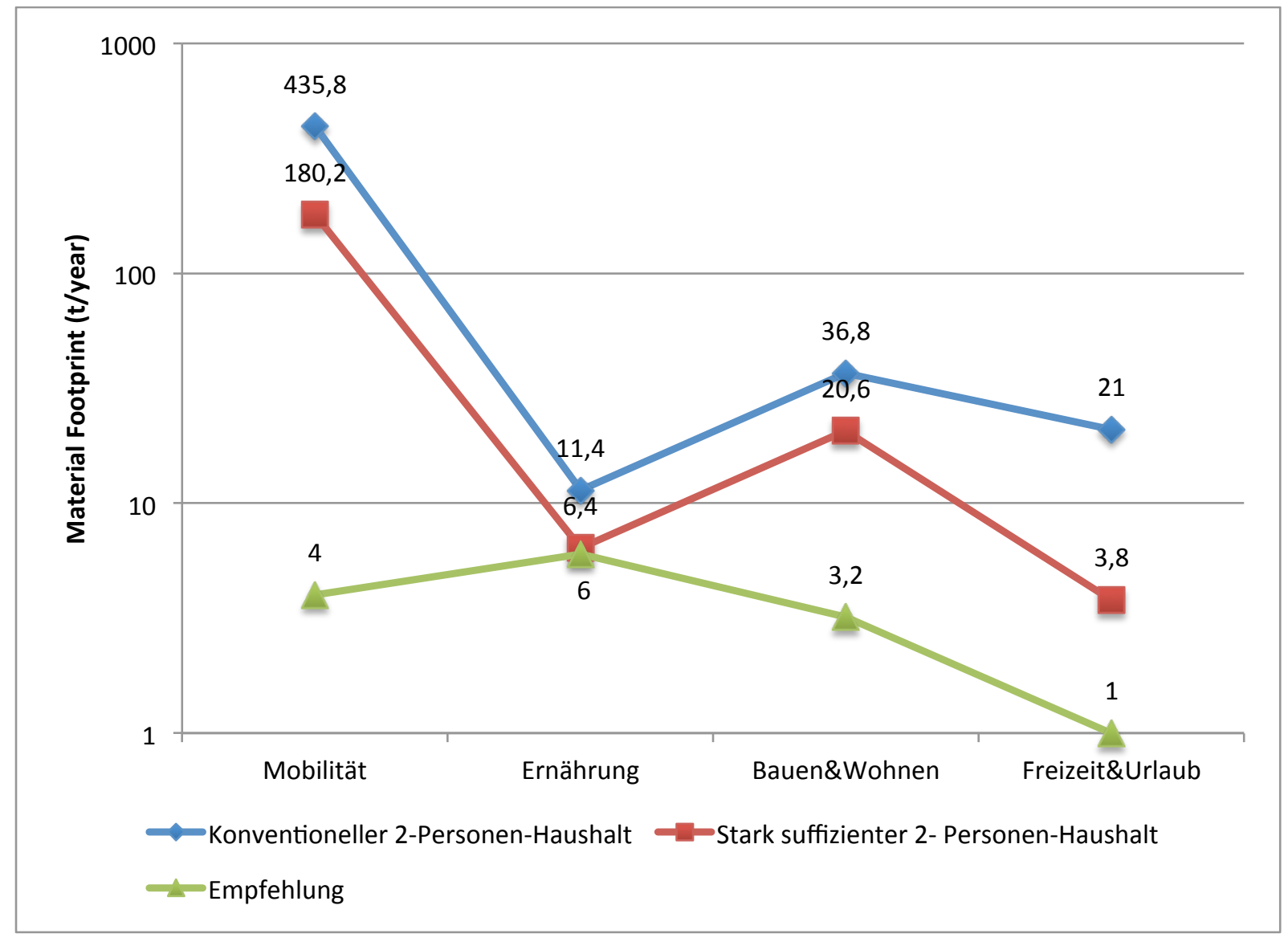

Abbildung 1: Darstellung der unterschiedlichen Ressourcenverbräuche - stark suffizient und konventioneller Haushalt im Vergleich zum Zielwert beschrieben von Lettenmeier et al. (2014) - in Material Footprint (t/Jahr) (eigene Berechnung - berechnet auf 2-Personen-Haushalt)

Die Abbildung 1 verdeutlicht die deutlichen Unterschiede der Haushalte, nicht nur im Vergleich miteinander, sondern insbesondere dann, wenn diese mit den von Lettenmeier et al. (2014) erarbeiteten Zielwert zu einem idealtypischen Ressourcenverbrauch pro Person (hier hochgerechnet auf 2-Personen-Haushalt) und Jahr verglichen werden. Im Rahmen dieser logarithmischen Einteilung kann gezeigt werden, dass der Zielwert für zwei Personen von 4 t/Jahr im Bereich Mobilität von den zwei konventionell handelnden Konsumenten um den Faktor 1300 überschritten wird. Selbst der suffizientere Personenhaushalt überschreitet die Empfehlung um den Faktor 10. Dies mag darauf zurückzuführen zu sein, dass der konventionell handelnde Haushalt über zwei Autos verfügt und viel mit dem PKW unterwegs ist. Pendlerstrecken mit dem eigenen PKW machen auch beim suffizienteren Haushalt den Hauptteil der Mobilitätsaufkommen aus. Darüber hinaus wird deutlich, dass der suffizientere Haushalt durch einen überwiegend vegetarischen Ernährungsstil fast an die Empfehlungen von Lettenmeier et al. (2014) herankommen, während der konventionelle Haushalt fast 
doppelt so viele Ressourcen verbraucht. Größere Abweichungen gibt es ebenfalls im Bereich Wohnen. So ein Ressourcenverbrauch wäre bspw. über eine urbane, mittelgroße Stadtwohnung zu erreichen. Beide Haushalte sind allerdings in ländlichen Gebieten angesiedelt. Extreme Abweichungen lassen sich wieder im Bereich Freizeit feststellen, denn hier macht die Urlaubsgestaltung einen großen Anteil aus. Während die suffizienteren Personen gerne mit Bahn und Mietauto zum Campen fahren und dabei meist in Deutschland oder angrenzenden Länder bleiben, setzen die konventionell handelnden Personen auf Fernreisen und Städtereisen, möglichst mehrmals im Jahr. So ergeben sich in allen Handlungsfeldern moderate bis massive Unterschiede - ein weiterer Indikator für die ökologische Relevanz einer suffizienteren Ausrichtung von Haushalten.

\section{Erkenntnisse - viel Potential und viele Barrieren}

Der vorliegende Beitrag unterstreicht die Position des privaten Haushalts als Untersuchungsbereich für die Suffizienzforschung. Es wird deutlich, dass Suffizienzstrategien im Alltag häufig auf veränderten Kulturtechniken beruhen und bis heute nicht vollkommen erschlossen sind. Entscheidungsmuster und Praktiken sind aber als ein wichtiger Baustein für das Verständnis von Suffizienz im Alltagshandeln einzuschätzen. Dazu kommt, der Einzelne trifft seine Entscheidungen fast ausschließlich im Zusammenspiel mit seinem sozialen Umfeld bzw. unter Berücksichtigung seiner Möglichkeiten und seiner Ausstattung. Somit ergibt sich an dieser Stelle eine hohe Komplexität, der bei nachfolgenden Untersuchungen erschlossen werden muss.

Interessanterweise wird deutlich, dass es bereits heute möglich ist, Suffizienzstrategien umzusetzen. Einige Interviewpersonen setzen bereits durch ihre individuelle Selbstbindung vielfältige suffizientere Handlungsmuster um, ohne diese selbst als suffizient einzuschätzen.

Gleichzeitig eröffnen sich unzählige Barrieren, die an dieser Stelle nur in Ansätzen skizziert wurden. Die Barrieren zeigen sich in Form von Zeit-, Freiheits-, Komfort-, Status- oder Gewohnheitsverlusten, welche von weniger suffizienz-orientierten Haushalten gefürchtet werden. Es erfolgt bei Suffizienzstrategien häufig eine Umlage der gefühlten Kosten, die das Individuum nicht auslagern kann, sondern für die Allgemeinheit trägt, ohne dass diese abstrakte Allgemeinheit es im direkten Rücklauf dankt. Diese Gegebenheit verhindert wiederum häufig eine Veränderung des Lebensstils. 
Ungeachtet dessen ist der Haushalt ein Experimentierfeld, in dem relevante Praktiken und Handlungsmuster im Suffizienzkontext eingeübt werden, wie z.B. die Zubereitung von Speisen oder das Reparieren von Gütern. Dieses Experimentierfeld ist weiter in den wissenschaftlichen und in den wirtschaftlichen Fokus zu rücken, denn hier werden suffizientere Praktiken geformt.

Nicht zuletzt wird deutlich, dass es Handlungsfelder gibt, die sich eventuell bereits einer höheren Wertschätzung bzw. Aufmerksamkeit mit Bezug auf ihre Umweltrelevanz erfreuen wie z.B. Mobilität und Ernährung. In diesen Handlungsfeldern wird bereits vielfach ökologisch- und sozialorientiert agiert. Im Vergleich dazu beschreibt Spaargaren (2011), dass in den Niederlanden ebenfalls in den Bereichen Mobilität und Ernährung sowie Wohnen bereits viele ausgeprägte umweltgerechte Nutzungsmuster in Haushalten zu beobachten seien, während die Bereiche Tourismus und Freizeit sowie Bekleidung noch in vielen Aspekten nicht unter sozial- oder umweltgerechten Kriterien vom Einzelnen betrachtet werden (Spaargaren, 2011). Auf der Grundlage der Interviews kann von einer ähnlichen Situation in Deutschland ausgegangen werden. Weitere Forschungsanstrengungen müssen hier allerdings noch mehr Klarheit bringen, denn gleichzeitig wird deutlich, dass diese sinnstiftenden Veränderungen in weniger suffizienz-affinen Privathaushalten nur schwerlich umgesetzt werden, meist aufgrund mental verankterter Idealvorstellungen von Komfort und Bequemlichkeit. Langfristig müssen diese Bilder und Ängste abgebaut werden. Motive wie Zeit-, Freiheits- und Gewohnheitsverluste, Status- und Versäumnisängste, Unbehagen vor sozialer Desintegration und fehlendes Wissen müssen noch mehr aufgegriffen und relativiert werden (u.a. Stengel, 2011), um Privatpersonen die Unlust, sich von einem erlangten Konsum- und Komfortniveau zu lösen, zu nehmen. Die persönlichen Nutzenfunktionen sind anzusprechen, anfallende Kosten sind zu minimieren (auch: Diekmann \& Preisendörfer, 1998) und dadadurch entstehen meist wie selbstverständlich veränderte Denk- und Handlungsschemata, wie diese, die in den suffizienz-affineren Personenhaushalten bereits gelebt werden.

Besonders wichtig erscheinen diese Erkenntnisse auch für die Integration neuer Geschäftsmodelle. Denn neue Kulturtechniken brauchen neue, flexiblere Beschaffungsmöglichkeiten und andere Untersützungsmechanismen. Langfristig sollte es wichtiger sein, Nutzern schnell und einfach Möglichkeiten des Tauschens, des Sharings oder anderer Dienstleistungen zur Verfügung zu stellen. Anstatt immer neue, innovative Produkte 
zu entwickeln, die viele Haushalte gar nicht benötigen, da die Ausstattung bereits heute ausreichend ist. Es erscheint durchaus denkbar, dass sich suffizientere Haushalte langfristig durchaus in eigenen Bereichen von klassischen Unternehmensleistungen emanzipieren könnten, infolge neuer Kulturtechniken.

\section{$\underline{\text { Fazit }}$}

Die Suffizienzstrategie kann im Rahmen einer nachhaltigen Entwicklung sehr vorteilhaft sein, da diese vom Einzelnen umsetzbar ist, ohne auf technologische oder gesamtgesellschaftliche Veränderungen warten $\mathrm{zu}$ müssen. Trotzdem darf der Einzelne nicht von Politik und Gesellschaft allein gelassen werden, da bis heute vielerlei Barrieren und Hürden bei der Umsetzung der Suffizienzstrategien im Alltag und Haushalt warten.

Der vorliegende Beitrag fokussiert deshalb den Haushalt als einen wichtigen Ort für die Ausprägung und Festlegung des Konsumhandelns. So kann herausgestellt werden, dass der Privathaushalt als Settings für eine suffizienz-orientierte Lebensweise eine maßgebende Rolle spielt. Diese Rolle wurde bisher aber noch nicht ausreichend in Wissenschaft und Forschung beleuchtet, und auch die Wirtschaft und Unternehmen haben bisher kaum die Potentiale von suffizienz-orientierten Konsumenten erkannt.

Im Rahmen der Untersuchung wird ebenfalls deutlich, dass Suffizienzstrategien in Form veränderter Kulturtechniken umzusetzen sind. Dieser Fakt sollte ebenfalls von Wissenschaft und Wirtschaft als Ankerpunkt genutzt werden. Neue Kulturtechniken üben sich nur dann ein, wenn gesellschaftliche Hemmnisse abgebaut werden und z.B. suffizientere Praktiken die etablierten Komfortlösungen darstellen und Zeit, Geld und ganz natürlich ökologische Ressourcen sparen. Unterstrichen wird diese Annahme durch die beispielhafte Berechnung der Ressourcenaufwendungen. Hier wird deutlich, wie viel Potential aktuell in den Haushalten vorhanden ist. Politik und Gesellschaft, als auch Unternehmen müssen umdenken und ihren Beitrag leisten, damit Suffizienz zur Normalität führen kann. Denn was der Einzelne z.B. im Geschäften kaufen, mieten oder leihen kann, verkörpert Normalität. 


\section{Literatur}

Bruppacher, S. (2008). Wie und warum eignen wir uns einen nachhaltigen Lebensstil an? Wissenschaft \& Umwelt, 11, 216-223.

Cherrier, H., \& Gurrieri, L. (2012). Anti-consumption Choices Performed in a Drinking Culture: Normative Struggles and Repairs. Journal of Macromarketing, Dec 6, 2012. DOI: $10.1177 / 0276146712467805$

Diekmann, A., \& Preisendörfer, P. (1998). Umweltbewusstsein und Umweltverhalten in Lowund High-Cost-Situationen. Zeitschrift Für Soziologie, 27(6), 438-453.

Götz, K., Glatzer, W., \& Gölz, S. (2011). Haushaltsproduktion und Stromverbrauch Möglichkeiten der Stromersparnis im privaten Haushalt. In R. Defila, D. G. Antonietta, \& R. Kaufmann-Hayoz (Eds.), Wesen und Wege nachhaltigen Konsums. München: Oekom. 265-282.

Kettschau, I., Methfessel, B., \& Piorkowsky, M.-B. (2000). Familie 2000. Hochgehren: Schneider Verlag.

Lettenmeier, M., Liedtke, C., \& Rohn, H. (2014). Eight Tonnes of Material Footprint Suggestion for a Resource Cap for Household Consumption in Finland. Resources 2014, 3(3), 488-515. doi:10.3390/resources3030488

Liedtke, C., Baedeker, C., Hasselkuß, M., Rohn, H., \& Grinewitschus, V. (2014). Userintegrated innovation in Sustainable LivingLabs: an experimental infrastructure for researching and developing sustainable product service systems. Journal of Cleaner Production. doi:10.1016/j.jclepro.2014.04.070

Piorkowsky, M.-B. (2000). Konsum aus Sicht der Haushaltsökonomik. In D. Rosenkranz \& N. F. Schneider (Eds.), Konsum. Soziologie, ökonomische und psychologische Perspektiven, Opladen: Leske und Budrich. 184-206.

Schlegel-Matthies, K., Methfessel, B., \& Bigga, R. (2009). Materielle Ressourcen - oder: Warum, wozu und wie haben und nutzen wir Güter? Haushalt \& Bildung, 86(2), 10-14.

Schulze, G. (2005). Erlebnisgesellschaft. Frankfurt am Main: Campus Verlag.

Spaargaren, G. (2011). Theories of practice: Agency, technology, and culture. Exploring the relevance of practice theories for the governance of sustainable consumption practices in the new world-order. Global Environmental Change, 21, 813-822. doi:10.1016/j.gloenvcha.2011.03.010

Spangenberg, J. H., \& Lorek, S. (2002). Environmentally sustainable household consumption : from aggregate environmental pressures to priority fields of action. Ecological Economics, 43, 127-140.

Stengel, O. (2011). Suffizienz - Die Konsumgesellschaft in der ökologischen Krise. München: Oekom. 
Strauss, A., \& Corbin, J. (1996). Grounded Theory - Grundlagen qualitativer Sozialforschung. Weinheim: Beltz. 